\title{
Article
}

\section{Explicit Identities for 3-Variable Degenerate Hermite Kampé de Fériet Polynomials and Differential Equation Derived from Generating Function}

\author{
Kyung-Won Hwang ${ }^{1}$, Young-Soo Seol ${ }^{1}$ and Cheon-Seoung Ryoo ${ }^{2, *}$ \\ 1 Department of Mathematics, Dong-A University, Busan 49315, Korea; khwang@dau.ac.kr (K.-W.H.); \\ prosul76@dau.ac.kr (Y.-S.S.) \\ 2 Department of Mathematics, Hannam University, Daejeon 34430, Korea \\ * Correspondence: ryoocs@hnu.kr
}

check for

updates

Citation: Hwang, K.-W.; Seol, Y.-S.; Ryoo, C.-S. Explicit Identities for 3-Variable Degenerate Hermite Kampé de Fériet Polynomials and Differential Equation Derived from Generating Function. Symmetry 2021, 13, 7. https://doi.org/10.3390/ sym 13010007

Received: 30 November 2020 Accepted: 11 December 2020 Published: 22 December 2020

Publisher's Note: MDPI stays neutral with regard to jurisdictional clai$\mathrm{ms}$ in published maps and institutional affiliations.

Copyright: (C) 2020 by the authors. Licensee MDPI, Basel, Switzerland. This article is an open access article distributed under the terms and conditions of the Creative Commons Attribution (CC BY) license (https:// creativecommons.org/licenses/by/ $4.0 /)$.

\begin{abstract}
We get the 3-variable degenerate Hermite Kampé de Fériet polynomials and get symmetric identities for 3-variable degenerate Hermite Kampé de Fériet polynomials. We make differential equations coming from the generating functions of degenerate Hermite Kampé de Fériet polynomials to get some identities for 3-variable degenerate Hermite Kampé de Fériet polynomials,. Finally, we study the structure and symmetry of pattern about the zeros of the 3-variable degenerate Hermite Kampé de Fériet equations.
\end{abstract}

Keywords: differential equations; symmetric identities; 3-variable degenerate Hermite Kampé de Fériet polynomials; complex zeros

\section{Introduction}

The classical Hermite numbers $H_{n}$ and polynomials $H_{n}(x)$ are usually defined by the generating functions

$$
e^{-t^{2}}=\sum_{n=0}^{\infty} H_{n} \frac{t^{n}}{n !}
$$

and

$$
e^{2 x t-t^{2}}=\sum_{n=0}^{\infty} H_{n}(x) \frac{t^{n}}{n !} .
$$

Clearly, $H_{n}=H_{n}(0)$.

These numbers and polynomials have been studied because of important roles in many areas of mathematics (see References [1,2]). The special polynomials of 3-variable give partial differential equations of physical phenomenon. Physical problems was expressed by the special functions of mathematical physics. We recall that the 3-variable Hermite polynomials $H_{n}(x, y, z)$ made by the generating function (see Reference [3])

$$
\sum_{n=0}^{\infty} H_{n}(x, y, z) \frac{t^{n}}{n !}=e^{x t+y t^{2}+z t^{3}}
$$

are solutions in the system of equations

$$
\begin{aligned}
& \frac{\partial}{\partial y} H_{n}(x, y, z)=\frac{\partial^{2}}{\partial x^{2}} H_{n}(x, y, z), \\
& \frac{\partial}{\partial z} H_{n}(x, y, z)=\frac{\partial^{3}}{\partial x^{3}} H_{n}(x, y, z), \\
& H_{n}(x, 0,0)=x^{n} .
\end{aligned}
$$


In particular, one has

$$
H_{n}(2 x,-1,0)=H_{n}(x) .
$$

Many researchers studied special numbers and polynomials because of importance (see References [1-7]). The degenerate Bernoulli, Euler, Genocchi and tangent polynomials were studied in several papers (see References [8-12]). Recently, researchers have studied the differential equations which are related to generating functions of special polynomials (see References [13-18]).

We construct the 3-variable degenerate Hermite Kampé de Fériet polynomials and get symmetric identities for 3-variable degenerate Hermite Kampé de Fériet polynomials. Finally, we study the distribution and symmetry of pattern of the roots of the 3-variable degenerate Hermite Kampé de Fériet polynomials Hermite equations.

We define the 3-variable degenerate Hermite Kampé de Fériet polynomials $\mathbf{H}_{n}(x, y, z \mid \mu)$ made by the generating function

$$
\mathfrak{F}(t, x, y, z \mid \mu)=\sum_{n=0}^{\infty} \mathbf{H}_{n}(x, y, z \mid \mu){\frac{t^{n}}{n !}}_{n}=(1+\mu)^{\frac{x t}{\mu}}(1+\mu)^{\frac{y t^{2}}{\mu}}(1+\mu)^{\frac{z t^{3}}{\mu}} .
$$

Since $(1+\mu)^{\frac{t}{\mu}} \rightarrow e^{t}$ as $\mu \rightarrow 0$, it is clear that (4) reduces to (3). If $\mu \rightarrow 0$ and $z=0$, Equation (4) is the generating function of the 2-variable Hermite polynomials $H_{n}(x, y, 0)$. Observe that Hermite polynomials $H_{n}(x, y, 0)$ with the 2-variable are the solution of the heat equation (see Reference [17])

$$
\frac{\partial}{\partial y} H_{n}(x, y, 0)=\frac{\partial^{2}}{\partial x^{2}} H_{n}(x, y, 0), \quad H_{n}(x, 0,0)=x^{n} .
$$

Theorem 1. For $n=0,1, \ldots$, the 3-variable degenerate Hermite Kampé de Fériet polynomials $\mathbf{H}_{n}(x, y, z \mid \mu)$ with the generating function (4) are the solution of the differential equation

$$
\begin{aligned}
& \left(3 z \frac{\partial^{2}}{\partial x \partial y}+2 y \frac{\partial^{2}}{\partial x^{2}}+x \frac{\log (1+\mu)}{\mu} \frac{\partial}{\partial x}-n \frac{\log (1+\mu)}{\mu}\right) \mathbf{H}_{n}(x, y, z \mid \mu)=0, \\
& \mathbf{H}_{n}(x, 0,0 \mid \mu)=\left(\frac{\log (1+\mu)}{\mu}\right)^{n} x^{n} . \\
& \mathbf{H}_{n}(0, y, 0 \mid \mu)= \begin{cases}\left(\frac{\log (1+\mu)}{\mu}\right)^{k} y^{k} \frac{(2 k) !}{k !}, & \text { if } n=2 k \\
0, & \text { otherwise }\end{cases} \\
& \mathbf{H}_{n}(0,0, z \mid \mu)= \begin{cases}\left(\frac{\log (1+\mu)}{\mu}\right)^{k} z^{k} \frac{(3 k) !}{k !}, & \text { if } n=3 k \\
0, & \text { otherwise }\end{cases}
\end{aligned}
$$

Proof. We see that

$$
\mathfrak{F}(t, x, y, z \mid \mu)=(1+\mu)^{\frac{x t}{\mu}}(1+\mu)^{\frac{y t^{2}}{\mu}}(1+\mu)^{\frac{z t^{3}}{\mu}}
$$

satisfies

$$
\frac{\partial \mathfrak{F}(t, x, y, z \mid \mu)}{\partial t}-\frac{\log (1+\mu)}{\mu}\left(x+2 y t+3 z t^{2}\right) \mathfrak{F}(t, x, y, z \mid \mu)=0 .
$$


By substituting the series (4) for $\mathfrak{F}(t, x, y, z \mid \mu)$, one obtains

$$
\begin{gathered}
\mathbf{H}_{n+1}(x, y, z \mid \mu)-x \frac{\log (1+\mu)}{\mu} \mathbf{H}_{n}(x, y, z \mid \mu)-2 y \frac{\log (1+\mu)}{\mu} \mathbf{H}_{n-1}(x, y, z \mid \mu) \\
-n(n-1) 3 z \frac{\log (1+\mu)}{\mu} \mathbf{H}_{n-2}(x, y, z \mid \mu)=0, n=2,3, \ldots
\end{gathered}
$$

We get a recurrence relation for 3-variable degenerate Hermite Kampé de Fériet polynomials and another recurrence relation which comes from

$$
\frac{\partial \mathfrak{F}(t, x, y, z \mid \mu)}{\partial x}-\frac{\log (1+\mu)}{\mu} t \mathfrak{F}(t, x, y, z \mid \mu)=0 .
$$

This implies

$$
\frac{\partial \mathbf{H}_{n}(x, y, z \mid \mu)}{\partial x}-n \frac{\log (1+\mu)}{\mu} \mathbf{H}_{n-1}(x, y, z \mid \mu)=0, n=1,2, \ldots
$$

On the other hand, since

$$
\frac{\partial \mathfrak{F}(t, x, y, z \mid \mu)}{\partial y}-\frac{\log (1+\mu)}{\mu} t^{2} \mathfrak{F}(t, x, y, z \mid \mu)=0,
$$

we get

$$
\frac{\partial \mathbf{H}_{n}(x, y, z \mid \mu)}{\partial y}-n(n-1) \frac{\log (1+\mu)}{\mu} \mathbf{H}_{n-2}(x, y, z \mid \mu)=0, n=2,3, \ldots
$$

Eliminate $\mathbf{H}_{n-1}(x, y, z \mid \mu)$ and $\mathbf{H}_{n-2}(x, y, z \mid \mu)$ from (5)-(7) to obtain

$$
\mathbf{H}_{n+1}(x, y, z \mid \mu)-x \frac{\log (1+\mu)}{\mu} \mathbf{H}_{n}(x, y, z \mid \mu)-2 y \frac{\partial \mathbf{H}_{n}(x, y, z \mid \mu)}{\partial x}-3 z \frac{\partial \mathbf{H}_{n}(x, y, z \mid \mu)}{\partial y}=0 .
$$

Differentiate this equation and use (6) again to get

$$
\begin{gathered}
3 z \frac{\partial^{2} \mathbf{H}_{n}(x, y, z \mid \mu)}{\partial x \partial y}+2 y \frac{\partial \mathbf{H}_{n}(x, y, z \mid \mu)}{\partial x}+x \frac{\log (1+\mu)}{\mu} \mathbf{H}_{n}(x, y, z \mid \mu) \\
-n \frac{\log (1+\mu)}{\mu} \mathbf{H}_{n}(x, y, z \mid \mu)=0,(n=0,1, \ldots),
\end{gathered}
$$

thus we have shown the theorem.

Theorem 2. The 3-variable degenerate Hermite Kampé de Fériet polynomials $\mathbf{H}_{n}(x, y, z \mid \mu)$ with the generating function (4) are the solution of the differential equation

$$
\begin{aligned}
& \left(3 z \frac{\mu}{\log (1+\mu)} \frac{\partial^{3}}{\partial x^{3}}+2 y \frac{\partial^{2}}{\partial x^{2}}+x \frac{\log (1+\mu)}{\mu} \frac{\partial}{\partial x}-n \frac{\log (1+\mu)}{\mu}\right) \mathbf{H}_{n}(x, y, z \mid \mu)=0, \\
& \mathbf{H}_{n}(x, 0,0 \mid \mu)=\left(\frac{\log (1+\mu)}{\mu}\right)^{n} x^{n}, n=0,1,2, \ldots \\
& \mathbf{H}_{n}(0, y, 0 \mid \mu)= \begin{cases}\left(\frac{\log (1+\mu)}{\mu}\right)^{k} y^{k} \frac{(2 k) !}{k !}, & \text { if } n=2 k \\
0, & \text { otherwise }\end{cases} \\
& \mathbf{H}_{n}(0,0, z \mid \mu)= \begin{cases}\left(\frac{\log (1+\mu)}{\mu}\right)^{k} z^{k} \frac{(3 k) !}{k !}, & \text { if } n=3 k \\
0, & \text { otherwise. }\end{cases}
\end{aligned}
$$


Proof. We get another recurrence relation which comes from

$$
\frac{\partial^{2} \mathfrak{F}(t, x, y, z \mid \mu)}{\partial x \partial y}-\left(\frac{\log (1+\mu)}{\mu}\right)^{2} t^{2} \mathfrak{F}(t, x, y, z \mid \mu)=0 .
$$

This implies

$$
\frac{\partial^{2} \mathbf{H}_{n}(x, y, z \mid \mu)}{\partial x \partial y}=n(n-1)(n-2)\left(\frac{\log (1+\mu)}{\mu}\right)^{2} \mathbf{H}_{n-3}(x, y, z \mid \mu)=0, n=3,4, \ldots
$$

Again, we also have

$$
\frac{\partial^{3} \mathfrak{F}(t, x, y, z \mid \mu)}{\partial x^{3}}-\left(\frac{\log (1+\mu)}{\mu}\right)^{3} t^{3} \mathfrak{F}(t, x, y, z \mid \mu)=0 .
$$

This implies

$$
\frac{\partial^{3} \mathbf{H}_{n}(x, y, z \mid \mu)}{\partial x^{3}}=n(n-1)(n-2)\left(\frac{\log (1+\mu)}{\mu}\right)^{3} \mathbf{H}_{n-3}(x, y, z \mid \mu)=0, n=3,4, \ldots
$$

Thus, from (8)-(10), the degenerate Hermite Kampé de Fériet polynomials $\mathbf{H}_{n}(x, y, z \mid \mu)$ of 3-variable with the generating function (4) are the solution of the differential equation

$$
\begin{aligned}
& \frac{3 z \mu}{\log (1+\mu)} \frac{\partial^{3} \mathbf{H}_{n}(x, y, z \mid \mu)}{\partial x^{3}}+2 y \frac{\partial^{2} \mathbf{H}_{n}(x, y, z \mid \mu)}{\partial x^{2}} \\
& \quad+x \frac{\log (1+\mu)}{\mu} \frac{\partial \mathbf{H}_{n}(x, y, z \mid \mu)}{\partial x}-n \frac{\log (1+\mu)}{\mu} \mathbf{H}_{n}(x, y, z \mid \mu)=0 .
\end{aligned}
$$

Therefore, we are done.

We see another application of the differential equation for $\mathbf{H}_{n}(x, y, z \mid \mu)$. The polynomials $\mathbf{H}_{n}(x, y, z \mid \mu)$ have this relations

$$
\begin{aligned}
& \frac{\partial \mathbf{H}_{n}(x, y, z \mid \mu)}{\partial y}-\frac{\mu}{\log (1+\mu)} \frac{\partial^{2} \mathbf{H}_{n}(x, y, z \mid \mu)}{\partial x^{2}}=0, \\
& \frac{\partial \mathbf{H}_{n}(x, y, z \mid \mu)}{\partial z}-\left(\frac{\mu}{\log (1+\mu)}\right)^{2} \frac{\partial^{2} \mathbf{H}_{n}(x, y, z \mid \mu)}{\partial x^{2}}=0,
\end{aligned}
$$

which in view of the initial condition are solved by

$$
\begin{aligned}
& \mathbf{H}_{n}(x, 0,0 \mid \mu)=\left(\frac{\log (1+\mu)}{\mu}\right)^{n} x^{n}, n=0,1,2, \ldots \\
& \mathbf{H}_{n}(0, y, 0 \mid \mu)= \begin{cases}\left(\frac{\log (1+\mu)}{\mu}\right)^{k} y^{k} \frac{(2 k) !}{k !}, & \text { if } n=2 k \\
0, & \text { otherwise }\end{cases} \\
& \mathbf{H}_{n}(0,0, z \mid \mu)= \begin{cases}\left(\frac{\log (1+\mu)}{\mu}\right)^{k} z^{k} \frac{(3 k) !}{k !}, & \text { if } n=3 k \\
0, & \text { otherwise }\end{cases}
\end{aligned}
$$

The Stirling numbers of the first kind, $S_{1}(n, k)$, were defined by (see References [8-10])

$$
(x)_{n}=\sum_{k=0}^{n} S_{1}(n, k) x^{k} .
$$


$S_{1}(n, k)$ is

$$
\sum_{n=m}^{\infty} S_{1}(n, m) \frac{t^{n}}{n !}=\frac{1}{m !}(\log (1+t))^{m}
$$

where $(x)_{n}=x(x-1) \cdots(x-n+1)$. We see the binomial theorem: for a variable $x$,

$$
\begin{aligned}
(1+\mu)^{\frac{x t}{\mu}} & =\sum_{m=0}^{\infty}\left(\frac{t x}{\mu}\right)_{m} \frac{\mu^{m}}{m !} \\
& =\sum_{m=0}^{\infty}\left(\sum_{l=0}^{m} S_{1}(m, l)\left(\frac{t x}{\mu}\right)^{l} \frac{\mu^{m}}{m !}\right) \\
& =\sum_{l=0}^{\infty}\left(\sum_{m=l}^{\infty} S_{1}(m, l) x^{l} \mu^{m-l} \frac{l !}{m !}\right) \frac{t^{l}}{l !} .
\end{aligned}
$$

By (4), we have

$$
\begin{aligned}
& \sum_{n=0}^{\infty} \mathbf{H}_{n}(x, y, z \mid \mu) \frac{t^{n}}{n !}=(1+\mu)^{\frac{x t}{\mu}}(1+\mu)^{\frac{y t^{2}}{\mu}}(1+\mu)^{\frac{z t^{2}}{\mu}} \\
& =\sum_{k=0}^{\infty}\left(\frac{y \log (1+\mu)}{\mu}\right)^{k} \frac{t^{2 k}}{k !} \sum_{l=0}^{\infty}\left(\frac{x \log (1+\mu)}{\mu}\right)^{l} \frac{t^{l}}{l !} \sum_{j=0}^{\infty}\left(\frac{x \log (1+\mu)}{\mu}\right)^{j} \frac{t^{3 j}}{j !} \\
& =\sum_{n=0}^{\infty}\left(\sum_{k=0}^{\left[\frac{n}{2}\right]}\left(\frac{\log (1+\mu)}{\mu}\right)^{n-k} y^{k} x^{n-2 k} \frac{n !}{k !(n-2 k) !}\right) \frac{t^{n}}{n !} \sum_{j=0}^{\infty}\left(\frac{x \log (1+\mu)}{\mu}\right)^{j} \frac{t^{3 j}}{j !} \\
& \sum_{n=0}^{\infty}\left(\sum_{k=0}^{\left[\frac{n}{3}\right]} \sum_{l=0}^{\left[\frac{n-3 k}{2}\right]}\left(\frac{\log (1+\mu)}{\mu}\right)^{n-2 k-l} \frac{z^{k} y^{l} x^{n-3 k-2 l} n !(n-3 k) !}{k ! l !(n-3 k) !(n-3 k-2 l) !}\right) \frac{t^{n}}{n !} .
\end{aligned}
$$

If we compare the coefficients $\frac{t^{n}}{n !}$ on the both sides of (12), we have representation of $\mathbf{H}_{n}(x, y, z \mid \mu)$.

$$
\mathbf{H}_{n}(x, y, z \mid \mu)=\sum_{k=0}^{\left[\frac{n}{3}\right]} \sum_{l=0}^{\left[\frac{n-3 k}{2}\right]}\left(\frac{\log (1+\mu)}{\mu}\right)^{n-2 k-l} \frac{z^{k} y^{l} x^{n-3 k-2 l} n !(n-3 k) !}{k ! l !(n-3 k) !(n-3 k-2 l) !},
$$

and [ ] denotes taking the integer part.

The following elementary properties of $\mathbf{H}_{n}(x, y, z \mid \mu)$ are deduced form (4). We delete the details. 
Theorem 3. For any positive n, we have
1. $\mathbf{H}_{n}(x, 0,0 \mid \mu)=\sum_{m=n}^{\infty} S_{1}(m, n) x^{n} \mu^{m-n} \frac{n !}{m !}$.
2. $\mathbf{H}_{n}\left(x_{1}+x_{2}, y, z \mid \mu\right)=\sum_{l=0}^{n} \sum_{m=l}^{\infty}\left(\begin{array}{l}n \\ l\end{array}\right) \mathbf{H}_{n-l}\left(x_{1}, y, z \mid \mu\right) S_{1}(m, l) x_{2}^{l} \mu^{m-l} \frac{l 1}{m !}$.
3. $\mathbf{H}_{n}\left(x, y_{1}+y_{2}, z \mid \mu\right)=\sum_{k=0}^{\left[\frac{n}{2}\right]} \mathbf{H}_{n-2 k}\left(x, y_{1}, z \mid \mu\right)\left(\frac{\log (1+\mu)}{\mu}\right)^{k} y_{2}^{k} \frac{n !}{k !(n-2 k) !}$.
4. $\quad \mathbf{H}_{n}\left(x, y_{1}+y_{2}, z \mid \mu\right)=\sum_{k=0}^{\left[\frac{n}{2}\right]} \sum_{m=k}^{\infty} \frac{S_{1}(m, k) y_{2}^{k} \mu^{m-k} n !}{m !(n-2 k) !} \mathbf{H}_{n-2 k}\left(x, y_{1}, z \mid \mu\right)$.
5. $\quad \mathbf{H}_{n}\left(x, y, z_{1}+z_{2} \mid \mu\right)=\sum_{k=0}^{\left[\frac{n}{3}\right]} \mathbf{H}_{n-3 k}\left(x, y, z_{1} \mid \mu\right)\left(\frac{\log (1+\mu)}{\mu}\right)^{k} z_{2}^{k} \frac{n !}{k !(n-3 k) !}$.
6. $\quad \mathbf{H}_{n}\left(x, y, z_{1}+z_{2} \mid \mu\right)=\sum_{k=0}^{\left[\frac{n}{3}\right]} \sum_{m=k}^{\infty} \frac{S_{1}(m, k) z_{2}^{k} \mu^{m-k} n !}{m !(n-3 k) !} \mathbf{H}_{n-3 k}\left(x, y, z_{1} \mid \mu\right)$.
7. $\mathbf{H}_{n}\left(x_{1}+x_{2}, y_{1}+y_{2}, z_{1}+z_{2} \mid \mu\right)=\sum_{l=0}^{n}\left(\begin{array}{l}n \\ l\end{array}\right) \mathbf{H}_{l}\left(x_{1}, y_{1}, z_{1} \mid \mu\right) \mathbf{H}_{n-l}\left(x_{2}, y_{2}, z_{2} \mid \mu\right)$.

The paper is written by this process: We make symmetric identities about 3-variable degenerate Hermite Kampé de Fériet polynomials in Section 2. We also get formulas of 3-variable degenerate Hermite Kampé de Fériet polynomials. We induce the differential equations getting from the generating function of 3-variable degenerate Hermite Kampé de Fériet polynomials in Section 3:

$$
\begin{array}{r}
\left(\frac{\partial}{\partial t}\right)^{N} \mathfrak{F}(t, x, y, z \mid \mu)-a_{0}(N, x, y, z \mid \mu) \mathfrak{F}(t, x, y, z \mid \mu)-a_{1}(N, x, y, z \mid \mu) t \mathfrak{F}(t, x, y, z \mid \mu)-\cdots \\
-a_{N-1}(N, x, y, z \mid \mu) t^{N-1} \mathfrak{F}(t, x, y, z \mid \mu)-a_{N}(N, x, y, z \mid \mu) t^{N} \mathfrak{F}(t, x, y, z \mid \mu)=0 .
\end{array}
$$

In Section 4, we study distribution of computer graphic about the roots of the 3variable degenerate Hermite Kampé de Fériet equation $\mathbf{H}_{n}(x, y, z \mid \mu)=0$. Finally, we see the symmetric pattern of the roots of polynomials $\mathbf{H}_{n}(x, y, z \mid \mu)=0$ and indicate some open problems.

\section{Symmetric Identities for the 3-Variable Degenerate Hermite Kampé de Fériet Polynomials}

In this section, we give symmetric identities for the 3-variable degenerate Hermite Kampé de Fériet polynomials. We also get some formulas and properties of the 3-variable degenerate Hermite Kampé de Fériet polynomials.

Theorem 4. Let $a, b>0$ and $a \neq b$. Then

$$
a^{m} \mathbf{H}_{m}\left(b x, b^{2} y, b^{3} z \mid \mu\right)=b^{m} \mathbf{H}_{m}\left(a x, a^{2} y, a^{3} z \mid \mu\right) .
$$

Proof. Let $a, b>0$ and $a \neq b$. We start with

$$
\mathcal{F}(t, x, y, z, \mu)=(1+\mu))^{\frac{a b x t}{\mu}}(1+\mu) \frac{a^{2} b^{2} y t^{2}}{\mu}(1+\mu) \frac{\frac{a^{3} b^{3} z t^{3}}{\mu}}{\mu} .
$$

Then the expression for $\mathcal{F}(t, x, y, z, \mu)$ is symmetric in $a$ and $b$

$$
\mathcal{F}(t, x, y, z, \mu)=\sum_{m=0}^{\infty} \mathbf{H}_{m}\left(a x, a^{2} y, a^{3} z \mid \mu\right) \frac{(b t)^{m}}{m !}=\sum_{m=0}^{\infty} b^{m} \mathbf{H}_{m}\left(a x, a^{2} y, a^{3} z \mid \mu\right) \frac{t^{m}}{m !}
$$


We can get that

$$
\mathcal{F}(t, x, y, z, \mu)=\sum_{m=0}^{\infty} \mathbf{H}_{m}\left(b x, b^{2} y, b^{3} z \mid \mu\right) \frac{(a t)^{m}}{m !}=\sum_{m=0}^{\infty} a^{m} \mathbf{H}_{m}\left(b x, b^{2} y, b^{3} z \mid \mu\right) \frac{t^{m}}{m !} .
$$

When we compare the coefficients of $\frac{t^{m}}{m !}$ on the right hand sides of the last two equations, the proof is completed.

When we let $\mu \rightarrow 0$ in Theorem 4 , we have the corollary

Corollary 1. Let $a, b>0$ and $a \neq b$. Then

$$
a^{m} H_{m}\left(b x, b^{2} y, b^{3} z\right)=b^{m} H_{m}\left(a x, a^{2} y, a^{3} z\right) .
$$

Again, we now use

$$
\mathcal{G}(t, x, y, z, \mu)=\frac{a b t(1+\mu)^{\frac{a b x t}{\mu}}(1+\mu)^{\frac{a^{2} b^{2} y t^{2}}{\mu}}(1+\mu)^{\frac{a^{3} b^{3} z t^{3}}{\mu}}\left((1+\mu)^{\frac{a b t}{\mu}}-1\right)}{\left((1+\mu)^{\frac{a t}{\mu}}-1\right)\left((1+\mu)^{\frac{b t}{\mu}}-1\right)} .
$$
function

For $\mu \in \mathbb{C}$, we define the degenerate Bernoulli polynomials related to the generating

$$
\sum_{n=0}^{\infty} \mathcal{B}_{n}(x \mid \mu) \frac{t^{n}}{n !}=\frac{t}{(1+\mu)^{\frac{t}{\mu}}-1}(1+\mu)^{\frac{x t}{\mu}}
$$

If we give $x=0, \mathcal{B}_{n}(\mu)=\mathcal{B}_{n}(0, \mu)$ are called the degenerate Bernoulli numbers. Let us look at few terms:

$$
\begin{aligned}
& \mathcal{B}_{0}(x \mid \mu)=\frac{\mu}{\log (1+\mu)^{\prime}} \\
& \mathcal{B}_{1}(x \mid \mu)=x-\frac{1}{2} \\
& \mathcal{B}_{2}(x \mid \mu)=\frac{x^{2} \log (1+\mu)}{\mu}-\frac{x \log (1+\mu)}{\mu}+\frac{\log (1+\mu)}{6 \mu}, \\
& \mathcal{B}_{3}(x \mid \mu)=\frac{x^{3} \log (1+\mu)^{2}}{\mu^{2}}-\frac{3 x^{2} \log (1+\mu)^{2}}{2 \mu^{2}}+\frac{x \log (1+\mu)^{2}}{2 \mu^{2}}, \\
& \mathcal{B}_{4}(x \mid \mu)=\frac{x^{4} \log (1+\mu)^{3}}{\mu^{3}}-\frac{2 x^{3} \log (1+\mu)^{3}}{\mu^{3}}+\frac{x^{2} \log (1+\mu)^{3}}{\mu^{3}}-\frac{\log (1+\mu)^{3}}{30 \mu^{3}} .
\end{aligned}
$$

Let each integer $k \geq 0 . S_{k}(n)=0^{k}+1^{k}+2^{k}+\cdots+(n-1)^{k}$ is called sum of integers. A generalized falling factorial sum $\sigma_{k}(n, \mu)$ can be defined by the generation function

$$
\sum_{k=0}^{\infty} \sigma_{k}(n, \mu) \frac{t^{k}}{k !}=\frac{(1+\mu) \frac{\frac{(n+1) t}{\mu}}{(1+\mu)^{\frac{t}{\mu}}-1} .}{.}
$$

We look at this $\lim _{\mu \rightarrow 0} \sigma_{k}(n, \mu)=S_{k}(n)$. 
Theorem 5. Let $a, b>0$ and $a \neq b$. Then

$$
\begin{aligned}
& \sum_{i=0}^{n} \sum_{m=0}^{i}\left(\begin{array}{c}
n \\
i
\end{array}\right)\left(\begin{array}{c}
i \\
m
\end{array}\right) a^{i} b^{n+1-i} \mathcal{B}_{m}(\mu) \mathbf{H}_{i-m}\left(b x, b^{2} y, b^{3} z \mid \mu\right) \sigma_{n-i}(a-1, \mu) \\
& =\sum_{i=0}^{n} \sum_{m=0}^{i}\left(\begin{array}{c}
n \\
i
\end{array}\right)\left(\begin{array}{c}
i \\
m
\end{array}\right) b^{i} a^{n+1-i} \mathcal{B}_{m}(\mu) \mathbf{H}_{i-m}\left(a x, a^{2} y, a^{3} z \mid \mu\right) \sigma_{n-i}(b-1, \mu) .
\end{aligned}
$$

Proof. From $\mathcal{G}(t, x, y, z, \mu)$, we get the following result:

$$
\begin{aligned}
& \mathcal{G}(t, x, y, z, \mu) \\
& =\frac{a b t(1+\mu) \frac{a b x t}{\mu}(1+\mu) \frac{a^{2} b^{2} y t^{2}}{\mu}(1+\mu) \frac{a^{3} b^{3} z t^{3}}{\mu}\left((1+\mu)^{\frac{a b t}{\mu}}-1\right)}{\left((1+\mu)^{\frac{a t}{\mu}}-1\right)\left((1+\mu)^{\frac{b t}{\mu}}-1\right)} \\
& =\frac{a b t}{\left((1+\mu)^{\frac{a t}{\mu}}-1\right)}(1+\mu) \frac{a b x t}{\mu}(1+\mu) \frac{a^{2} b^{2} y t^{2}}{\mu}(1+\mu) \frac{a^{3} b^{3} z t^{3}}{\mu} \frac{\left.(1+\mu)^{\frac{a b t}{\mu}}-1\right)}{\left((1+\mu)^{\frac{b t}{\mu}}-1\right)} \\
& =b \sum_{n=0}^{\infty} \mathcal{B}_{n}(\mu) \frac{(a t)^{n}}{n !} \sum_{n=0}^{\infty} \mathbf{H}_{n}\left(b x, b^{2} y, b^{3} z \mid \mu\right) \frac{(a t)^{n}}{n !} \sum_{n=0}^{\infty} \sigma_{k}(a-1, \mu) \frac{(b t)^{n}}{n !} \\
& =\sum_{n=0}^{\infty}\left(\sum_{i=0}^{n} \sum_{m=0}^{i}\left(\begin{array}{c}
n \\
i
\end{array}\right)\left(\begin{array}{c}
i \\
m
\end{array}\right) a^{i} b^{n+1-i} \mathcal{B}_{m}(\mu) \mathbf{H}_{i-m}\left(b x, b^{2} y, b^{3} z \mid \mu\right) \sigma_{n-i}(a-1, \mu)\right) \frac{t^{n}}{n !} \text {. }
\end{aligned}
$$

If we follow a similar way, we have

$$
\begin{aligned}
& \mathcal{G}(t, x, y, z, \mu) \\
& =\frac{a b t}{\left(\begin{array}{c}
(1+\mu)^{\frac{b t}{\mu}}-1 \\
(1+\mu)
\end{array}\right)} \\
& =a \sum_{n=0}^{\infty} \mathcal{B}_{n}(\mu) \frac{(b t)^{n}}{n !} \sum_{n=0}^{\infty} \mathbf{H}_{n}\left(a x, a^{2} y, a^{3} z \mid \mu\right) \frac{a^{2} b^{2} y t^{2}}{\mu}(1+\mu) \frac{a^{3} b^{3} z t^{3}}{\mu !} \sum_{n=0}^{\infty} \sigma_{k}(a-1, \mu) \frac{(a t)^{n}}{n !} \\
& =\sum_{n=0}^{\infty}\left(\sum_{i=0}^{n} \sum_{m=0}^{i}\left(\begin{array}{c}
n \\
i
\end{array}\right)\left(\begin{array}{c}
i \\
m
\end{array}\right) b^{i} a^{n+1-i} \mathcal{B}_{m}(\mu) \mathbf{H}_{i-m}\left(a x, a^{2} y, a^{3} z \mid \mu\right) \sigma_{n-i}(b-1, \mu)\right) \frac{\left.\frac{a t}{\mu}-1\right)}{n !}
\end{aligned}
$$

If we compare the coefficients of $\frac{t^{n}}{n !}$ on the right hand sides of the last two equations, then the proof is completed.

If we give $\mu \rightarrow 0$ in Theorem 5 , we have the corollary 
Corollary 2. Let $a, b>0$ and $a \neq b$. Then

$$
\begin{aligned}
& \sum_{i=0}^{n} \sum_{m=0}^{i}\left(\begin{array}{c}
n \\
i
\end{array}\right)\left(\begin{array}{c}
i \\
m
\end{array}\right) a^{i} b^{n+1-i} B_{m} H_{i-m}\left(b x, b^{2} y, b^{3} z\right) S_{n-i}(a-1) \\
& =\sum_{i=0}^{n} \sum_{m=0}^{i}\left(\begin{array}{c}
n \\
i
\end{array}\right)\left(\begin{array}{c}
i \\
m
\end{array}\right) b^{i} a^{n+1-i} B_{m} H_{i-m}\left(a x, a^{2} y, a^{3} z\right) S_{n-i}(b-1),
\end{aligned}
$$

where $B_{m}$ are Bernoulli numbers (see References [8-10]).

Theorem 6. Let $m, n, N$ be nonnegative integers. Then,

$$
\begin{aligned}
& \sum_{k=0}^{m}\left(\begin{array}{c}
m \\
k
\end{array}\right)(-n)^{m-k}\left(\frac{\log (1+\mu)}{\mu}\right)^{m-k} \mathbf{H}_{N+k}(x, y, z \mid \mu) \\
& =\sum_{k=0}^{N}\left(\begin{array}{l}
N \\
k
\end{array}\right) n^{N-k}\left(\frac{\log (1+\mu)}{\mu}\right)^{N-k} \mathbf{H}_{m+k}\left(x-n\left(\frac{\log (1+\mu)}{\mu}\right), y, z \mid \mu\right) .
\end{aligned}
$$

Proof. If we take $N$-th many derivative with respect to $t$ in (4), we have

$$
\begin{aligned}
\left(\frac{\partial}{\partial t}\right)^{N} \mathfrak{F}(t, x, y, z \mid \mu) & =\left(\frac{\partial}{\partial t}\right)^{N}(1+\mu)^{\frac{x t}{\mu}}(1+\mu)^{\frac{y t^{2}}{\mu}}(1+\mu)^{\frac{z t^{3}}{\mu}} \\
& =\sum_{m=0}^{\infty} \mathbf{H}_{m+N}(x, y, z \mid \mu) \frac{t^{m}}{m !} .
\end{aligned}
$$

If we use the Cauchy product and multiplying the exponential series $e^{x t}=\sum_{m=0}^{\infty} x^{m} \frac{t^{m}}{m !}$ on both sides of (13), we get

$$
\begin{aligned}
& e^{-n\left(\frac{\log (1+\mu)}{\mu}\right) t}\left(\frac{\partial}{\partial t}\right)^{N} \mathfrak{F}(t, x, y, z \mid \mu) \\
& =\left(\sum_{m=0}^{\infty}(-n)^{m}\left(\frac{\log (1+\mu)}{\mu}\right)^{m} \frac{t^{m}}{m !}\right)\left(\sum_{m=0}^{\infty} \mathbf{H}_{m+N}(x, y, z \mid \mu) \frac{t^{m}}{m !}\right) \\
& =\sum_{m=0}^{\infty}\left(\sum_{k=0}^{m}\left(\begin{array}{c}
m \\
k
\end{array}\right)(-n)^{m-k}\left(\frac{\log (1+\mu)}{\mu}\right)^{m-k} \mathbf{H}_{N+k}(x, y, z \mid \mu)\right) \frac{t^{m}}{m !} .
\end{aligned}
$$

When we use (14) and the Leibniz rule, we have

$$
\begin{aligned}
& e^{-n\left(\frac{\log (1+\mu)}{\mu}\right) t}\left(\frac{\partial}{\partial t}\right)^{N} \mathfrak{F}(t, x, y, z \mid \mu) \\
& =\sum_{k=0}^{N}\left(\begin{array}{c}
N \\
k
\end{array}\right) n^{N-k}\left(\frac{\log (1+\mu)}{\mu}\right)^{N-k}\left(\frac{\partial}{\partial t}\right)^{k}\left(e^{-n\left(\frac{\log (1+\mu)}{\mu}\right) t} \mathfrak{F}(t, x, y, z \mid \mu)\right) \\
& =\sum_{m=0}^{\infty}\left(\sum_{k=0}^{N}\left(\begin{array}{c}
N \\
k
\end{array}\right) n^{N-k}\left(\frac{\log (1+\mu)}{\mu}\right)^{N-k} \mathbf{H}_{m+k}\left(x-n\left(\frac{\log (1+\mu)}{\mu}\right), y, z \mid \mu\right)\right) \frac{t^{m}}{m !} .
\end{aligned}
$$
pleted.

If we use (14) and (15), and compare the coefficients of $\frac{t^{m}}{m !}$, then the proof is com- 
If we plug in $m=0$ in (15), then we obtain the following theorem

Theorem 7. For $N=0,1,2, \ldots$, we have

$$
\mathbf{H}_{N}(x, y, z \mid \mu)=\sum_{k=0}^{N}\left(\begin{array}{l}
N \\
k
\end{array}\right) n^{N-k}\left(\frac{\log (1+\mu)}{\mu}\right)^{N-k} \mathbf{H}_{k}\left(x-n\left(\frac{\log (1+\mu)}{\mu}\right), y, z \mid \mu\right) .
$$

\section{Differential Equations Related to 3-Variable Degenerate Hermite Kampé de Fériet Polynomials}

Many researchers have studied differential equations which are related to the generating functions of special numbers and polynomials in References [13-18] in order to make formulas for special numbers and polynomials. Recall that

$$
\begin{aligned}
\mathfrak{F}=\mathfrak{F}(t, x, y, z \mid \mu) & =(1+\mu)^{\frac{x t}{\mu}}(1+\mu)^{\frac{y t^{2}}{\mu}}(1+\mu)^{\frac{z t^{3}}{\mu}} \\
& =\sum_{n=0}^{\infty} \mathbf{H}_{n}(x, y, z \mid \mu) \frac{t^{n}}{n !}, \quad \mu, x, t \in \mathbb{C} .
\end{aligned}
$$

In this section, we study the differential equations with coefficients $a_{i}(N, x, y, z \mid \mu)$ coming from the generating functions of the 3-variable degenerate Hermite Kampé de Fériet polynomials:

$$
\left(\frac{\partial}{\partial t}\right)^{N} \mathfrak{F}(t, x, y, z \mid \mu)-a_{0}(N, x, y, z \mid \mu) \mathfrak{F}(t, x, y, z \mid \mu)-\cdots-a_{N}(N, x, y, z \mid \mu) t^{N} \mathfrak{F}(t, x, y, z \mid \mu)=0 .
$$

From (16), it follows

$$
\begin{aligned}
\mathfrak{F}^{(1)} & =\frac{\partial}{\partial t} \mathfrak{F}(t, x, y, \mu) \\
& =\frac{\partial}{\partial t}\left((1+\mu)^{\frac{x t}{\mu}}(1+\mu)^{\frac{y t^{2}}{\mu}}(1+\mu)^{\frac{z t^{3}}{\mu}}\right) \\
& =\left(\frac{\left(x+2 y t+3 z t^{2}\right) \log (1+\mu)}{\mu}\right)(1+\mu)^{\frac{x t}{\mu}}(1+\mu)^{\frac{y t^{2}}{\mu}}(1+\mu)^{\frac{z t^{3}}{\mu}} \\
& =\left(\frac{\left(x+2 y t+3 z t^{2}\right) \log (1+\mu)}{\mu}\right) \mathfrak{F}(t, x, y, z \mid \mu),
\end{aligned}
$$

$$
\begin{aligned}
\mathfrak{F}^{(2)}= & \frac{\partial}{\partial t} \mathfrak{F}^{(1)}(t, x, y, z \mid \mu) \\
= & \left(\frac{(2 y+6 z t) \log (1+\mu)}{\mu}\right) \mathfrak{F}(t, x, y, z \mid \mu)+\left(\frac{\left(x+2 y t+3 z t^{2}\right) \log (1+\mu)}{\mu}\right) \mathfrak{F}^{(1)}(t, x, y, z \mid \mu) \\
= & \left(\left(\frac{\log (1+\mu)}{\mu}\right) 2 y+\left(\frac{\log (1+\mu)}{\mu}\right)^{2} x^{2}\right) \mathfrak{F}(t, x, y, z \mid \mu) \\
& +\left(\left(\frac{\log (1+\mu)}{\mu}\right) 6 z+\left(\frac{\log (1+\mu)}{\mu}\right)^{2} 4 x y\right) t \mathfrak{F}(t, x, y, z \mid \mu) \\
& +\left(\left(\frac{\log (1+\mu)}{\mu}\right)^{2}\left(4 y^{2}+6 x z\right)\right) t^{2} \mathfrak{F}(t, x, y, z \mid \mu) \\
& +\left(\left(\frac{\log (1+\mu)}{\mu}\right)^{2} 12 y z\right) t^{3} \mathfrak{F}(t, x, y, z \mid \mu) \\
& +\left(\left(\frac{\log (1+\mu)}{\mu}\right)^{2}(3 z)^{2}\right) t^{2} \mathfrak{F}(t, x, y, z \mid \mu) .
\end{aligned}
$$


When we continue this process, we can guess that

$$
\begin{aligned}
\mathfrak{F}^{(N)} & =\left(\frac{\partial}{\partial t}\right)^{N} \mathfrak{F}(t, x, y, z \mid \mu) \\
& =\sum_{i=0}^{2 N} a_{i}(N, x, y, z \mid \mu) t^{i} \mathfrak{F}(t, x, y, z \mid \mu),(N=0,1,2, \ldots)
\end{aligned}
$$

If we differentiate (19) with respect to $t$, we have

$$
\begin{aligned}
& \mathfrak{F}^{(N+1)}=\frac{\partial \mathfrak{F}^{(N)}}{\partial t}=\sum_{i=0}^{2 N} a_{i}(N, x, y, z \mid \mu) i t^{i-1} \mathfrak{F}(t, x, y, z \mid \mu) \\
& +\sum_{i=0}^{2 N} a_{i}(N, x, y, z \mid \mu) t^{i} \mathfrak{F}^{(1)}(t, x, y, z \mid \mu) \\
& =\sum_{i=0}^{2 N}(i) a_{i}(N, x, y, z \mid \mu) t^{i-1} \mathfrak{F}(t, x, y, z \mid \mu) \\
& +\sum_{i=0}^{2 N} \frac{x \log (1+\mu)}{\mu} a_{i}(N, x, y, z \mid \mu) t^{i} \mathfrak{F}(t, x, y, z \mid \mu) \\
& +\sum_{i=0}^{2 N} \frac{2 y \log (1+\mu)}{\mu} a_{i}(N, x, y, z \mid \mu) t^{i+1} \mathfrak{F}(t, x, y, z \mid \mu) \\
& +\sum_{i=0}^{2 N} \frac{3 z \log (1+\mu)}{\mu} a_{i-1}(N, x, y, z \mid \mu) t^{i+2} \mathfrak{F}(t, x, y, z \mid \mu) \\
& =\sum_{i=0}^{2 N-1}(i+1) a_{i+1}(N, x, y, z \mid \mu) t^{i} F(t, x, y, z \mid \mu) \\
& +\sum_{i=0}^{2 N} \frac{x \log (1+\mu)}{\mu} a_{i}(N, x, y, z \mid \mu) t^{i} \mathfrak{F}(t, x, y, z \mid \mu) \\
& +\sum_{i=1}^{2 N+1} \frac{2 y \log (1+\mu)}{\mu} a_{i-1}(N, x, y, z \mid \mu) t^{i} \mathfrak{F}(t, x, y, z \mid \mu) \\
& +\sum_{i=2}^{2 N+2} \frac{3 z \log (1+\mu)}{\mu} a_{i-2}(N, x, y, z \mid \mu) t^{i} \mathfrak{F}(t, x, y, z \mid \mu) .
\end{aligned}
$$

Now we plug in $N+1$ instead of $N$ in (19) to find

$$
\mathfrak{F}^{(2 N+2)}=\sum_{i=0}^{N+1} a_{i}(N+1, x, y, z \mid \mu) t^{i} \mathfrak{F}(t, x, y, z \mid \mu) .
$$


Comparing the coefficients from (20) and (21), we get

$$
\begin{aligned}
& a_{0}(N+1, x, y, z \mid \mu)=a_{1}(N, x, y, z \mid \mu)+\frac{x \log (1+\mu)}{\mu} a_{0}(N, x, y, z \mid \mu), \\
& a_{1}(N+1, x, y, z \mid \mu)=2 a_{2}(N, x, y, z \mid \mu)+\frac{x \log (1+\mu)}{\mu} a_{1}(N, x, y, z \mid \mu) \\
& +\frac{2 y \log (1+\mu)}{\mu} a_{0}(N, x, y, z \mid \mu), \\
& a_{2 N}(N+1, x, y, z \mid \mu)=\frac{x \log (1+\mu)}{\mu} a_{2 N}(N, x, y, z \mid \mu) \\
& +\frac{2 y \log (1+\mu)}{\mu} a_{2 N-1}(N, x, y, z \mid \mu)+\frac{3 z \log (1+\mu)}{\mu} a_{2 N-2}(N, x, y, z \mid \mu), \\
& a_{2 N+1}(N+1, x, y, z \mid \mu)=\frac{2 y \log (1+\mu)}{\mu} a_{N}(N, x, y, z \mid \mu) \\
& +\frac{3 z \log (1+\mu)}{\mu} a_{2 N-1}(N, x, y, z \mid \mu), \\
& a_{2 N+2}(N+1, x, y, z \mid \mu)=\frac{3 z \log (1+\mu)}{\mu} a_{2 N}(N, x, y, z \mid \mu),
\end{aligned}
$$

and

$$
\begin{aligned}
a_{i}(N+1, x, y, z \mid \mu) & =(i+1) a_{i+1}(N, x, y, z \mid \mu) \\
& +\frac{x \log (1+\mu)}{\mu} a_{i}(N, x, y, z \mid \mu) \\
& +\frac{2 y \log (1+\mu)}{\mu} a_{i-1}(N, x, y, z \mid \mu) \\
& +\frac{3 z \log (1+\mu)}{\mu} a_{i-2}(N, x, y, z \mid \mu),(2 \leq i \leq 2 N-1) .
\end{aligned}
$$

In addition, by (16), we have

$$
\mathfrak{F}(t, x, y, z \mid \mu)=\mathfrak{F}^{(0)}(t, x, y, z \mid \mu)=a_{0}(0, x, y, z \mid \mu) \mathfrak{F}(t, x, y, z \mid \mu) .
$$

By (24), we get

$$
a_{0}(0, x, y, z \mid \mu)=1 \text {. }
$$

It is easy to show that

$$
\begin{aligned}
& \frac{x \log (1+\mu)}{\mu} \mathfrak{F}(t, x, y, z \mid \mu)+\frac{2 y \log (1+\mu)}{\mu} t \mathfrak{F}(t, x, y, z \mid \mu) \\
& \quad+\frac{2 z \log (1+\mu)}{\mu} t^{2} \mathfrak{F}(t, x, y, z \mid \mu) \\
& =\mathfrak{F}^{(1)}(t, x, y, z \mid \mu) \\
& =\sum_{i=0}^{2} a_{i}(1, x, y, z \mid \mu) t^{i} \mathfrak{F}(t, x, y, z \mid \mu) \\
& =a_{0}(1, x, y, z \mid \mu) \mathfrak{F}(t, x, y, z \mid \mu)+a_{1}(1, x, y, z \mid \mu) t \mathfrak{F}(t, x, y, z \mid \mu) \\
& \quad+a_{2}(1, x, y, z \mid \mu) t^{2} \mathfrak{F}(t, x, y, z \mid \mu) .
\end{aligned}
$$


Thus, by (26), we also get

$$
\begin{aligned}
& a_{0}(1, x, y, \mu)=\frac{x \log (1+\mu)}{\mu}, \\
& a_{1}(1, x, y, z \mid \mu)=\frac{2 y \log (1+\mu)}{\mu}, \\
& a_{2}(1, x, y, z \mid \mu)=\frac{3 z \log (1+\mu)}{\mu} .
\end{aligned}
$$

From (22), we note that

$$
\begin{aligned}
& a_{0}(N+1, x, y, z \mid \mu)=a_{1}(N, x, y, z \mid \mu)+\frac{x \log (1+\mu)}{\mu} a_{0}(N, x, y, z \mid \mu), \\
& a_{0}(N, x, y, z \mid \mu)=a_{1}(N-1, x, y, z \mid \mu)+\frac{x \log (1+\mu)}{\mu} a_{0}(N-1, x, y, z \mid \mu), \\
& a_{0}(N+1, x, y, z \mid \mu)=\sum_{i=0}^{N}\left(\frac{x \log (1+\mu)}{\mu}\right)^{i} a_{1}(N-i, x, y, z \mid \mu) \\
& +\left(\frac{\log (1+\mu)}{\mu}\right)^{N+1} x^{N+1},
\end{aligned}
$$

For $i=1$, we have

$$
\begin{aligned}
a_{1}(N+1, x, y, z \mid \mu) & =2 \sum_{k=0}^{N}\left(\frac{x \log (1+\mu)}{\mu}\right)^{k} a_{2}(N-k, x, y, z \mid \mu) \\
& +\frac{2 y \log (1+\mu)}{\mu} \sum_{k=0}^{N}\left(\frac{x \log (1+\mu)}{\mu}\right)^{k} a_{0}(N-k, x, y, z \mid \mu),
\end{aligned}
$$

Continuing this process, we can deduce that, for $2 \leq i \leq 2 N-1$,

$$
\begin{aligned}
a_{i}(N+1, x, y, z \mid \mu)= & (i+1) \sum_{k=0}^{N}\left(\frac{x \log (1+\mu)}{\mu}\right)^{k} a_{i+1}(N-k, x, y, z \mid \mu) \\
& +\frac{2 y \log (1+\mu)}{\mu} \sum_{k=0}^{N}\left(\frac{x \log (1+\mu)}{\mu}\right)^{k} a_{i-1}(N-k, x, y, z \mid \mu) \\
& +\frac{3 z \log (1+\mu)}{\mu} \sum_{k=0}^{N}\left(\frac{x \log (1+\mu)}{\mu}\right)^{k} a_{i-2}(N-k, x, y, z \mid \mu) .
\end{aligned}
$$


For $i=2 N$, we get

$$
\begin{aligned}
& a_{2 N}(N+1, x, y, z \mid \mu)=\frac{x \log (1+\mu)}{\mu} a_{2 N}(N, x, y, z \mid \mu) \\
& \quad+\frac{2 y \log (1+\mu)}{\mu} a_{2 N-1}(N, x, y, z \mid \mu)+\frac{3 z \log (1+\mu)}{\mu} a_{2 N-2}(N, x, y, z \mid \mu), \\
& a_{2 N-2}(N, x, y, z \mid \mu)=\frac{x \log (1+\mu)}{\mu} a_{2 N-2}(N-1, x, y, z \mid \mu) \\
& \quad+\frac{2 y \log (1+\mu)}{\mu} a_{2 N-1}(N-1, x, y, z \mid \mu)+\frac{3 z \log (1+\mu)}{\mu} a_{2 N-4}(N-1, x, y, z \mid \mu), \\
& \ldots, \quad \\
& a_{2 N}(N+1, x, y, z \mid \mu)=\frac{x \log (1+\mu)}{\mu} \sum_{k=0}^{N}\left(\frac{3 z \log (1+\mu)}{\mu}\right)^{k} a_{2 N-2 k}(N-k, x, y, z \mid \mu) \\
& \quad+\frac{2 y \log (1+\mu)}{\mu} \sum_{k=0}^{N-1}\left(\frac{3 z \log (1+\mu)}{\mu}\right)^{k} a_{2 N-2 k-1}(N-k, x, y, z \mid \mu) .
\end{aligned}
$$

For $i=2 N+1$, we obtain

$$
\begin{aligned}
& a_{2 N+1}(N+1, x, y, z \mid \mu) \\
& \quad=\left(\frac{2 y \log (1+\mu)}{\mu}\right)^{k} \sum_{k=0}^{N}\left(\frac{3 z \log (1+\mu)}{\mu}\right)^{k} a_{2 N-2 k}(N-k, x, y, z \mid \mu) .
\end{aligned}
$$

For $i=2 N+2$, we have

$$
a_{2 N+2}(N+1, x, y, z \mid \mu)=\left(\frac{3 z \log (1+\mu)}{\mu}\right)^{N+1} .
$$

As a matrix, $a_{i}(j, x, y, z \mid \mu)_{0 \leq i \leq 2 N+2,0 \leq j \leq N+1}$ is given by

$$
\left(\begin{array}{ccccc}
1 & \frac{x \log (1+\mu)}{\mu} & \left(\frac{x \log (1+\mu)}{\mu}\right)^{2}+\frac{2 y \log (1+\mu)}{\mu} & \ldots & \\
0 & \frac{2 y \log (1+\mu)}{\mu} & \frac{6 z \log (1+\mu)}{\mu}+\left(\frac{\log (1+\mu)}{\mu}\right)^{2} 4 x y & \ldots & . \\
0 & \frac{3 z \log (1+\mu)}{\mu} & \left(\frac{2 y \log (1+\mu)}{\mu}\right)^{2}+\left(\frac{\log (1+\mu)}{\mu}\right)^{2} 6 x z & \ldots & \\
0 & 0 & \left(\frac{\log (1+\mu)}{\mu}\right)^{2} 12 y z & \ldots & \\
0 & 0 & \left(\frac{3 z \log (1+\mu)}{\mu}\right)^{2} & \ldots & \\
\vdots & \vdots & \vdots & \ddots & \\
0 & 0 & 0 & \ldots & \left(\frac{3 z \log (1+\mu)}{\mu}\right)^{N+1}
\end{array}\right)
$$

Therefore, by (28)-(33), we get the following theorem: 
Theorem 8. Let $N=0,1,2, \ldots$ The differential equation

$$
\left(\frac{\partial}{\partial t}\right)^{N} \mathfrak{F}(t, x, y, z \mid \mu)-\left(\sum_{i=0}^{N} a_{i}(N, x, y, z \mid \mu) t^{i}\right) \mathfrak{F}(t, x, y, z \mid \mu)=0
$$

has a solution

$$
\mathfrak{F}=\mathfrak{F}(t, x, y, z \mid \mu)=(1+\mu)^{\frac{x t}{\mu}}(1+\mu)^{\frac{y t^{2}}{\mu}}(1+\mu)^{\frac{z t^{3}}{\mu}}
$$

where

$$
\begin{aligned}
& a_{0}(N+1, x, y, z \mid \mu)=\sum_{i=0}^{N}\left(\frac{x \log (1+\mu)}{\mu}\right)^{i} a_{1}(N-i, x, y, z \mid \mu) \\
& +\left(\frac{\log (1+\mu)}{\mu}\right)^{N+1} x^{N+1} \\
& a_{1}(N+1, x, y, z \mid \mu)=2 \sum_{k=0}^{N}\left(\frac{x \log (1+\mu)}{\mu}\right)^{k} a_{2}(N-k, x, y, z \mid \mu) \\
& +\frac{2 y \log (1+\mu)}{\mu} \sum_{k=0}^{N}\left(\frac{x \log (1+\mu)}{\mu}\right)^{k} a_{0}(N-k, x, y, z \mid \mu) \\
& a_{2 N}(N+1, x, y, z \mid \mu)=\frac{x \log (1+\mu)}{\mu} \sum_{k=0}^{N}\left(\frac{3 z \log (1+\mu)}{\mu}\right)^{k} a_{2 N-2 k}(N-k, x, y, z \mid \mu) \\
& +\frac{2 y \log (1+\mu)}{\mu} \sum_{k=0}^{N-1}\left(\frac{3 z \log (1+\mu)}{\mu}\right)^{k} a_{2 N-2 k-1}(N-k, x, y, z \mid \mu) \\
& a_{2 N+1}(N+1, x, y, z \mid \mu) \\
& =\left(\frac{2 y \log (1+\mu)}{\mu}\right)^{k} \sum_{k=0}^{N}\left(\frac{3 z \log (1+\mu)}{\mu}\right)^{k} a_{2 N-2 k}(N-k, x, y, z \mid \mu), \\
& a_{2 N+2}(N+1, x, y, z \mid \mu)=\left(\frac{3 z \log (1+\mu)}{\mu}\right)^{N+1}, \\
& a_{i}(N+1, x, y, z \mid \mu)=(i+1) \sum_{k=0}^{N}\left(\frac{x \log (1+\mu)}{\mu}\right)^{k} a_{i+1}(N-k, x, y, z \mid \mu) \\
& +\frac{2 y \log (1+\mu)}{\mu} \sum_{k=0}^{N}\left(\frac{x \log (1+\mu)}{\mu}\right)^{k} a_{i-1}(N-k, x, y, z \mid \mu) \\
& +\frac{3 z \log (1+\mu)}{\mu} \sum_{k=0}^{N}\left(\frac{x \log (1+\mu)}{\mu}\right)^{k} a_{i-2}(N-k, x, y, z \mid \mu),(2 \leq i \leq 2 N-1) \text {. }
\end{aligned}
$$

We have a picture of the surface for this solution.

In Figure $1 \mathrm{a}$, we choose $-1 \leq x \leq 1,-1 \leq t \leq 1, \mu=1 / 3$, and $y=2, z=1$. In Figure $1 \mathrm{~b}$, we choose $-2 \leq y \leq 2,-1 \leq t \leq 1, \mu=1 / 3$, and $x=5, z=3$.

When we take $N$-th many derivative with respect to $t$ for (4), we have

$$
\left(\frac{\partial}{\partial t}\right)^{N} \mathfrak{F}(t, x, y, z \mid \mu)=\sum_{m=0}^{\infty} \mathbf{H}_{m+N}(x, y, z \mid \mu) \frac{t^{m}}{m !}
$$

From (19) and (34), we have the following theorem: 
Theorem 9. For $N=0,1,2, \ldots$, one obtains

$$
\mathbf{H}_{m+N}(x, y, z \mid \mu)=\sum_{i=0}^{m} \frac{\mathbf{H}_{m-i}(x, y, z \mid \mu) a_{i}(N, x, y, z \mid \mu) m !}{(m-i) !} .
$$

From (35) with $m=0$ one obtains the following corollary:

Corollary 3. For $N=0,1,2, \ldots$, one obtains

$$
\mathbf{H}_{N}(x, y, z \mid \mu)=a_{0}(N, x, y, z \mid \mu),
$$

where

$$
\begin{aligned}
a_{0}(N+1, x, y, z \mid \mu)= & 1 \\
a_{0}(N+1, x, y, z \mid \mu)= & \sum_{i=0}^{N}\left(\frac{x \log (1+\mu)}{\mu}\right)^{i} a_{1}(N-i, x, y, z \mid \mu) \\
& +\left(\frac{\log (1+\mu)}{\mu}\right)^{N+1} x^{N+1} .
\end{aligned}
$$

The first 3-variable degenerate Hermite Kampé de Feŕiet polynomials read

$$
\begin{aligned}
& \mathbf{H}_{0}(x, y, z \mid \mu)=1 \text {, } \\
& \mathbf{H}_{1}(x, y, z \mid \mu)=\frac{x \log (1+\mu)}{\mu} \\
& \mathbf{H}_{2}(x, y, z \mid \mu)=\frac{x^{2}(\log (1+\mu))^{2}}{\mu^{2}}+\frac{2 y \log (1+\mu)}{\mu} \\
& \mathbf{H}_{3}(x, y, z \mid \mu)=\frac{x^{3}(\log (1+\mu))^{3}}{\mu^{3}}+\frac{6 x y(\log (1+\mu))^{2}}{\mu^{2}}+\frac{6 z(\log (1+\mu))^{2}}{\mu^{2}}, \\
& \mathbf{H}_{4}(x, y, z \mid \mu)=\frac{x^{4}(\log (1+\mu))^{4}}{\mu^{4}}+\frac{12 x^{2} y(\log (1+\mu))^{3}}{\mu^{3}}+\frac{24 x z(\log (1+\mu))^{2}}{\mu^{2}} \\
& +\frac{12 y^{2}(\log (1+\mu))^{2}}{\mu^{2}} \\
& \mathbf{H}_{5}(x, y, z \mid \mu)=\frac{x^{5}(\log (1+\mu))^{5}}{\mu^{5}}+\frac{20 x^{3} y(\log (1+\mu))^{4}}{\mu^{4}}+\frac{60 x^{2} z(\log (1+\mu))^{3}}{\mu^{3}}+ \\
& +\frac{60 x y^{2}(\log (1+\mu))^{3}}{\mu^{3}}+\frac{120 y z(\log (1+\mu))^{2}}{\mu^{2}} .
\end{aligned}
$$



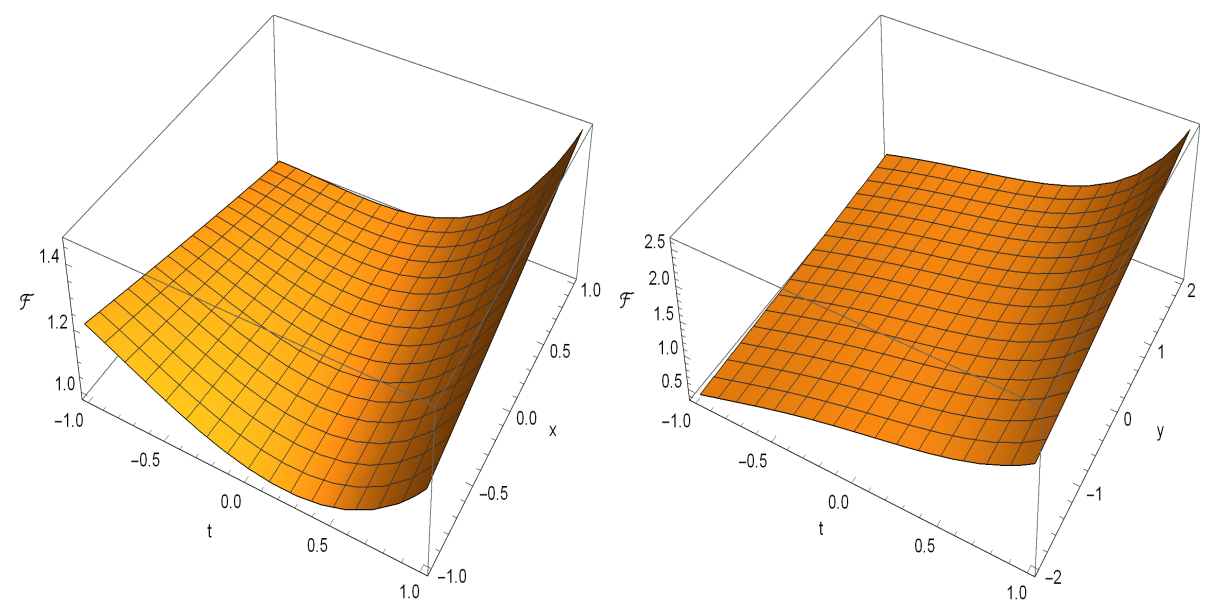

(a) $\mu=1 / 3, y=2$, and $z=1$

(b) $\mu=1 / 3, x=5$, and $z=3$

Figure 1. The surface for the solution $\mathfrak{F}(t, x, y, z \mid \mu)$.

\section{Roots of the 3-Variable Degenerate Hermite Kampé de Fériet Equations}

In this section we give a theoretical prediction via numerical experiments by finding a regular pattern for the roots of the 3-variable degenerate Hermite Kampé de Fériet equations $\mathbf{H}_{n}(x, y, z \mid \mu)=0$. To do this, we examine examples of several cases.

We look for the roots of $\mathbf{H}_{n}(x, y, z \mid \mu)=0$ for $n=40, y=2,-2,2+i,-2-i, z=$ $2,-2,2+i,-2-i, \mu=1 / 3$, and $x \in \mathbb{C}$ (Figure 2).

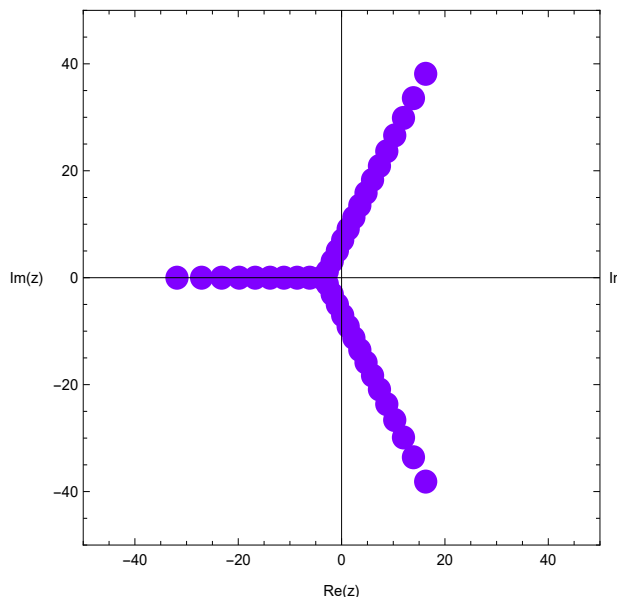

(a) $n=40, y=2$, and $z=2$

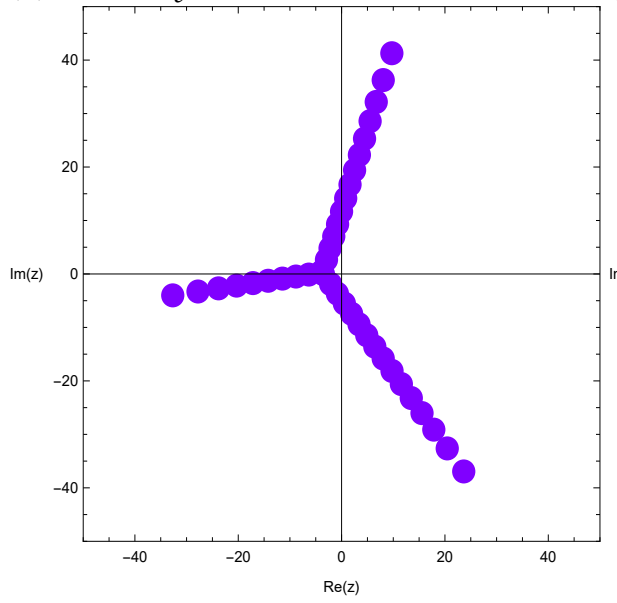

(c) $n=40, y=2+i$, and $z=2+i$

Figure 2. Zeros of $\mathbf{H}_{n}(x, y, z \mid \mu)=0$

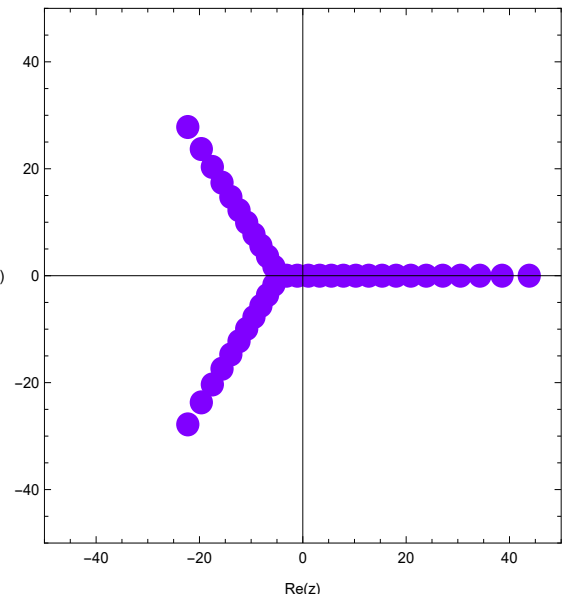

(b) $n=40, y=-2$, and $z=-2$

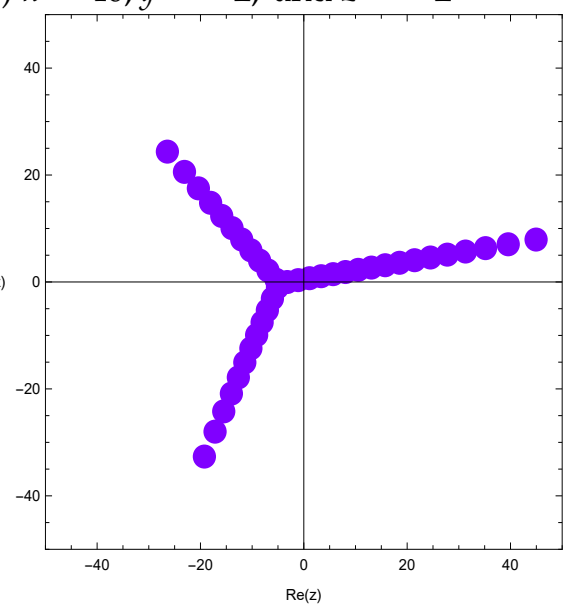

(d) $n=40, y=-2-i$, and $z=-2-i$ 
In Figure 2a, we select $n=40, y=2$, and $z=2$. In Figure $2 b$, we select $n=40$, $y=-2$, and $z=-2$. In Figure $2 \mathrm{c}$, we select $n=40, y=2+i$, and $z=2+i$. In Figure $2 \mathrm{~d}$, we select $n=40, y=-2-i$, and $z=-2-i$. A picture of the roots of the 3-variable degenerate Hermite Kampé de Fériet equation $\mathbf{H}_{n}(x, y, z \mid \mu)=0$ for $1 \leq n \leq 40, \mu=1 / 3$ from a 3-D structure are shown in Figure 3.

In Figure $3 a$, we select $y=2$ and $z=2$. In Figure $3 b$, we select $y=-2$ and $z=-2$. In Figure $3 c$, we select $y=2+i$ and $z=2+i$. In Figure $3 d$, we select $y=-2-i$ and $z=-2-i$. Our distributions for approximated solutions of real roots of equation $\mathbf{H}_{n}(x, y, z \mid \mu)=0$ are shown in Tables 1 and 2 .

We can observe a regular pattern of the complex roots of the 3-variable degenerate Hermite Kampé de Fériet equation $\mathbf{H}_{n}(x, y, z \mid \mu)=0$. We hope to prove regular pattern of the complex roots of the 3-variable degenerate Hermite Kampé de Fériet equations $\mathbf{H}_{n}(x, y, z \mid \mu)=0$ (Table 1$)$.

A picture of real roots of equations $\mathbf{H}_{n}(x, y, z \mid \mu)=0$ for $1 \leq n \leq 40, \mu=1 / 3$ are displayed in Figure 4.

Table 1. Numbers of real and complex zeros of $\mathbf{H}_{n}(x, y, z \mid \mu)=0$.

\begin{tabular}{ccccc}
\hline \multirow{2}{*}{ Degree $n$} & \multicolumn{2}{c}{$\boldsymbol{y}=\mathbf{2}, z=\mathbf{2}, \boldsymbol{\mu}=\mathbf{1 / 3}$} & \multicolumn{2}{c}{$\boldsymbol{y}=\mathbf{- 2}-\boldsymbol{z}=\mathbf{- 2}-\boldsymbol{i}=\mathbf{1 / 3}$} \\
\cline { 2 - 5 } & Real Zeros & Complex Zeros & Real Zeros & Complex Zeros \\
\hline 1 & 1 & 0 & 1 & 0 \\
\hline 2 & 0 & 2 & 0 & 2 \\
\hline 3 & 1 & 2 & 0 & 3 \\
\hline 4 & 0 & 4 & 0 & 4 \\
\hline 5 & 1 & 4 & 0 & 5 \\
\hline 6 & 2 & 4 & 0 & 6 \\
\hline 7 & 1 & 6 & 0 & 7 \\
\hline 8 & 2 & 6 & 0 & 9 \\
\hline 9 & 1 & 8 & 0 & 10 \\
\hline 10 & 2 & 8 & 0 & 8 \\
\hline
\end{tabular}

In Figure $4 a$, we select $y=2$ and $z=2$. In Figure $4 b$, we select $y=2$ and $z=-2$. In Figure $4 c$, we select $y=-2$ and $z=2$. In Figure $4 d$, we select $y=-2$ and $z=-2$.

Next, we obtain an approximate solution satisfying $\mathbf{H}_{n}(x, y, z \mid \mu)=0, x \in \mathbb{C}$ for given $n, y=2, z=2$, and $\mu=1 / 3$ in the Table 2 . 
Table 2. Approximate roots of $\mathbf{H}_{n}(x, y, z \mid \mu)=0, x \in \mathbb{C}, y=2, z=2$, and $\mu=1 / 3$.

\begin{tabular}{|c|c|}
\hline Degree $n$ & $x$ \\
\hline 1 & 0 \\
\hline 2 & $-2.1528 i, \quad 2.1528 i$ \\
\hline 3 & $-1.0705, \quad 0.5352+3.8424 i, \quad 0.5352-3.8424 i$ \\
\hline 4 & $\begin{array}{ccc}-1.1238-0.9119 i, & -1.1238+0.9119 i, & 1.1238+5.4316 i \\
& 1.1238-5.4316 i\end{array}$ \\
\hline 5 & $\begin{array}{c}-2.6937, \quad-0.3500-2.3168 i, \quad-0.3500+2.3168 i \\
1.6969+6.9000 i, \quad 1.6969-6.9000 i\end{array}$ \\
\hline 6 & $\begin{aligned}-3.6974, & -1.1016, \quad 0.1447-3.6924 i \\
0.1447+3.6924 i, & 2.2548+8.2725 i, \quad 2.2548-8.2725 i\end{aligned}$ \\
\hline 7 & $\begin{array}{c}-4.8260, \quad-1.0340+1.3912 i, \quad-1.0340-1.3912 i, \quad 0.6498+4.9794 i \\
0.6498-4.9794 i, \quad 2.7972-9.5687 i, \quad 2.7972+9.5687 i\end{array}$ \\
\hline
\end{tabular}
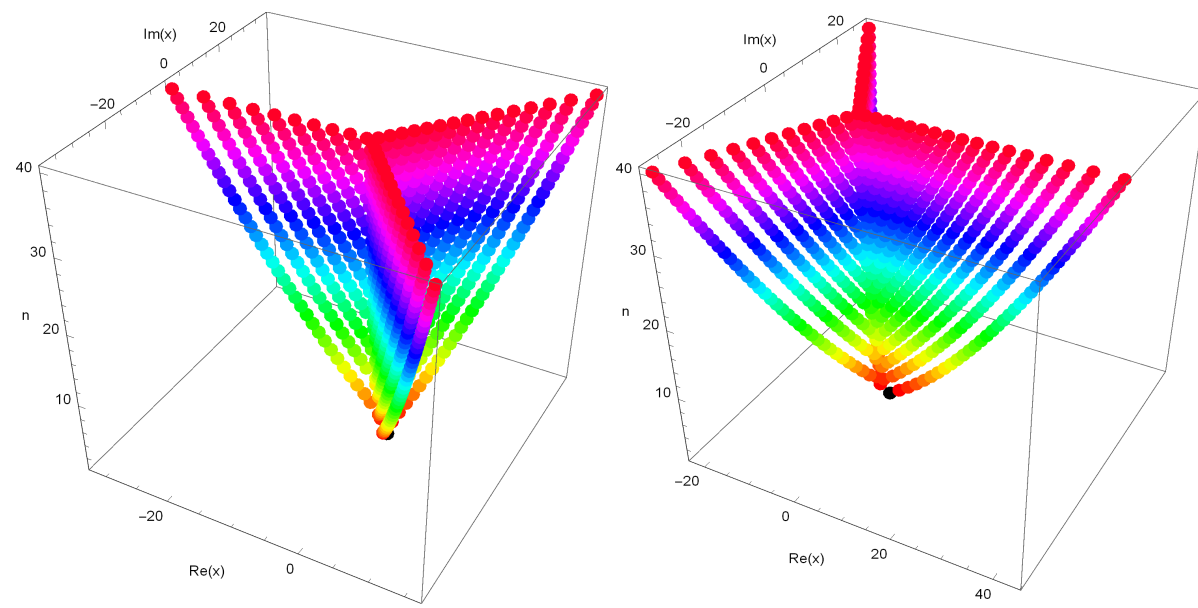

(a) $y=2$ and $z=2$

(b) $y=-2$ and $z=-2$

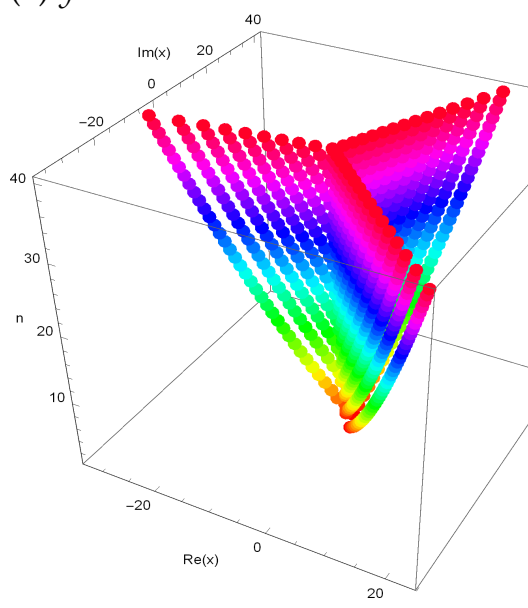

(c) $y=2+i$ and $z=2+i$

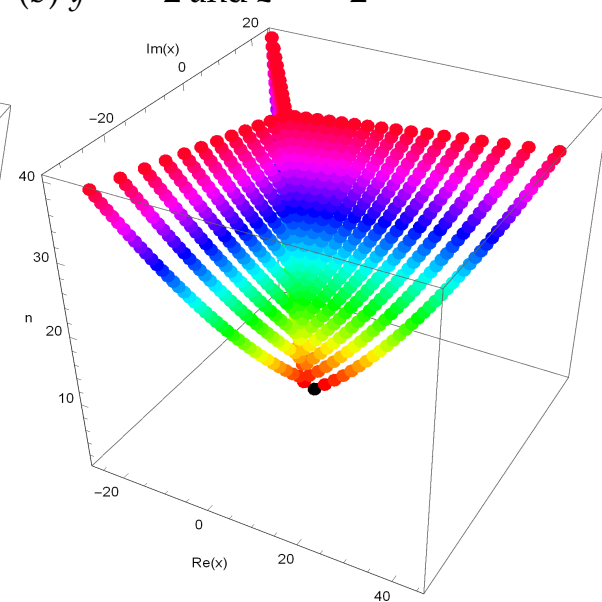

(d) $y=-2-i$, and $z=-2-i$

Figure 3. Stacks of zeros of $\mathbf{H}_{n}(x, y, z \mid \mu)=0,1 \leq n \leq 40$. 


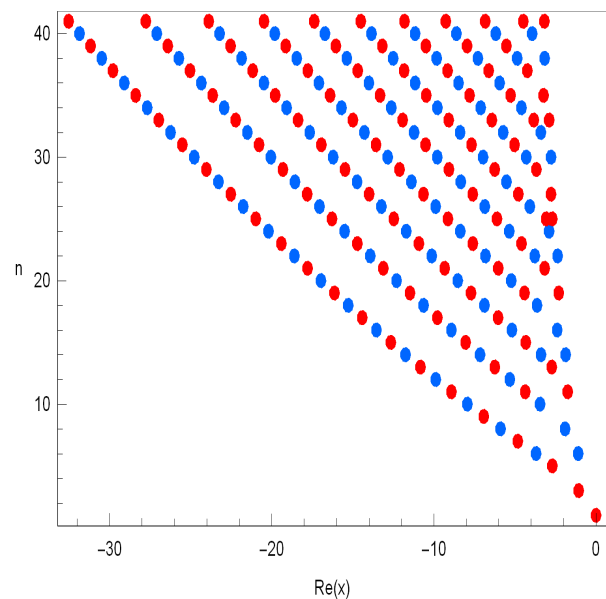

(a) $y=2$ and $z=2$

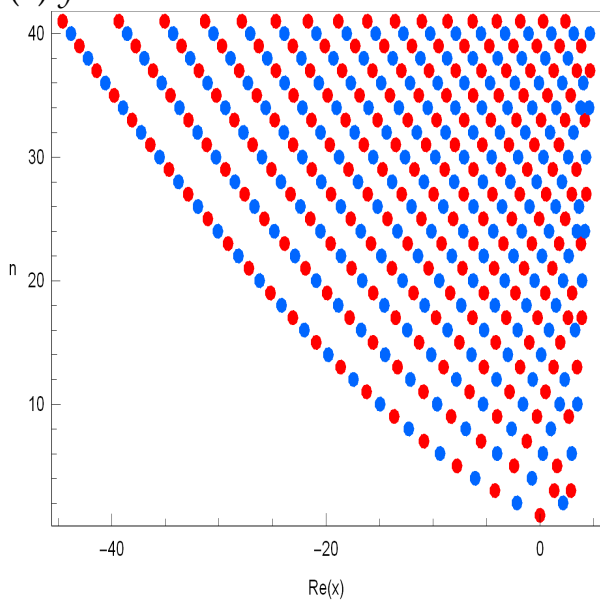

(c) $y=-2$ and $z=2$

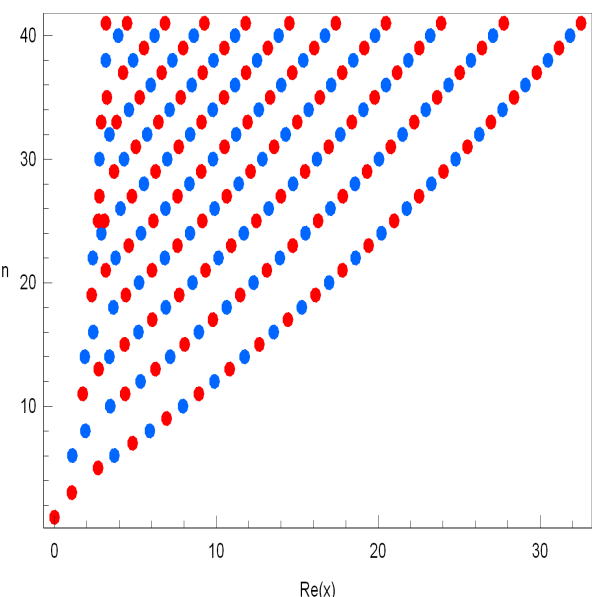

(b) $y=2$ and $z=-2$

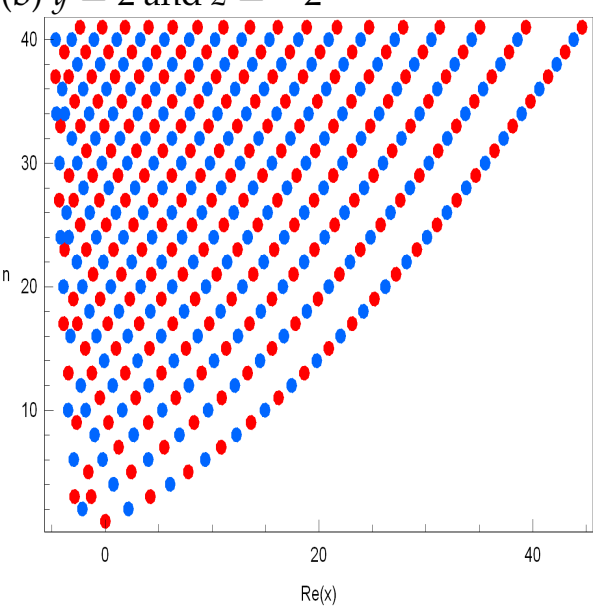

(d) $y=-2$ and $z=-2$

Figure 4. Real zeros of $\mathbf{H}_{n}(x, y, z \mid \mu)=0$ for $1 \leq n \leq 40$.

\section{Conclusions and Future Directions}

In this article, we constructed the 3-variable degenerate Hermite Kampé de Fériet polynomials and got symmetric identities for 3-variable degenerate Hermite Kampé de Fériet polynomials. We also made the differential equations which are related to the generating function of $\mathbf{H}_{n}(x, y, z \mid \mu)$. We also studied the symmetry of pattern of the roots of the 3-variable degenerate Hermite Kampé de Fériet equations $\mathbf{H}_{n}(x, y, z \mid \mu)=0$ for various variables $x, y$, and $z$. As a result, we found that the distribution of the roots of 3-variable degenerate Hermite Kampé de Fériet equations $\mathbf{H}_{n}(x, y, z \mid \mu)=0$ has regular pattern. So, we make the following series of conjectures with numerical experiments:

We use some notations. $R_{\mathbf{H}_{n}(x, y, z \mid \mu)}$ denotes the number of real zeros of $\mathbf{H}_{n}(x, y, z \mid \mu)=$ 0 on the real plane, that is, $\operatorname{Im}(x)=0$, and $C_{\mathbf{H}_{n}(x, y, z \mid \mu)}$ denotes the number of complex zeros of $\mathbf{H}_{n}(x, y, z \mid \mu)=0$. Since $n$ is the degree of the polynomial $\mathbf{H}_{n}(x, y, z \mid \mu)$, we obtaine $R_{\mathbf{H}_{n}(x, y, z \mid \mu)}=n-C_{\mathbf{H}_{n}(x, y, z \mid \mu)}$.

We can see a regular pattern of the complex roots of the 3-variable degenerate Hermite Kampé de Fériet equations $\mathbf{H}_{n}(x, y, z \mid \mu)=0$ for $y, z$, and $\mu$. Therefore, we can make thebelow conjecture.

Conjecture 1. Let $n=2,3, \ldots$, and $y \in \mathbb{C}$. Prove or disprove that

$$
R_{\mathbf{H}_{n}(x, y, z \mid \mu)}=0, \quad C_{\mathbf{H}_{n}(x, y, z \mid \mu)}=n,
$$

where $\mathbb{C}$ is the set of complex numbers. 
Conjecture 2. For $n=2,3, \ldots$, and $z \in \mathbb{C}$, prove or disprove that

$$
R_{\mathbf{H}_{n}(x, y, z \mid \mu)}=0, \quad C_{\mathbf{H}_{n}(x, y, z \mid \mu)}=n \text {. }
$$

The Conjectures 1 and 2 are unsolved problems for all variables $y, z$ and $\mu$.

We see that the solutions of the 3-variable degenerate Hermite Kampé de Fériet equations $\mathbf{H}_{n}(x, y, z \mid \mu)=0$ does not show reflection symmetry about $\operatorname{Re}(x)=a$ for $a \in \mathbb{R}$ (see Figures 2-4).

Conjecture 3. Prove that $\mathbf{H}_{n}(x, y, z \mid \mu), x \in \mathbb{C}, y, z \in \mathbb{R}$ as an analytic complex function has reflection symmetry $\operatorname{Im}(x)=0$.

Finally, we consider the more general problems. How many roots does $\mathbf{H}_{n}(x, y, z \mid \mu)=$ 0 have? We are not able to decide whether $\mathbf{H}_{n}(x, y, z \mid \mu)=0$ has $n$ distinct solutions. We would like to know the number of complex roots $C_{\mathbf{H}_{n}(x, y, z \mid \mu)}$ of $\mathbf{H}_{n}(x, y, z \mid \mu)=0$.

Conjecture 4. Prove or disprove that $\mathbf{H}_{n}(x, y, z \mid \mu)=0$ has $n$ distinct solutions.

The conjecture 4 is unsolved problem for all variables $n$ (see Tables 1 and 2).

If we can theoretically prove the above problems by drawing new ideas from various numerical results, we look forward to contributing to research related to the 3-variable degenerate Hermite Kampé de Fériet equations $\mathbf{H}_{n}(x, y, z \mid \mu)=0$ in applied mathematics, mathematical physics, and engineering.

Author Contributions: C.-S.R. and K-W.H. did typing together; C.-S.R. and Y.-S.S. draw pictures by using computor. K.-W.H., C.-S.R., and Y.-S.S. made Section 1. Y.-S.S. made Section 2. K.-W.H. made Section 3. C.-S.R. made Section 4. These authors contributed equally to this work. All authors have read and agreed to the published version of the manuscript.

Funding: This work was supported by the Dong-A university research fund.

Acknowledgments: The authors would like to thank the referees for their valuable comments, which improved the original manuscript in its present form.

Conflicts of Interest: The authors declare no conflict of interest.

\section{References}

1. Andrews, L.C. Special Functions for Engineers and Mathematicians; Macmillan. Co.: New York, NY, USA, 1985.

2. Appell, P.; de Fériet, J.H.K. Fonctions Hypergéométriques et Hypersphériques: Polynomes d Hermite; Gauthier-Villars: Paris, France, 1926.

3. Dattoli, G. Generalized Polynomials Operational Identities and Their Applications. J. Comput. Appl. Math. 2000, 118, 111-123. [CrossRef]

4. $\quad$ Erdelyi, A.; Magnus, W.; Oberhettinger, F.; Tricomi, F.G. Higher Transcendental Functions; Krieger: New York, NY, USA, 1981; Volume 3.

5. Andrews, G.E.; Askey, R.; Roy, R. Special Functions; Cambridge University Press: Cambridge, UK, 1999.

6. Arfken, G. Mathematical Methods for Physicists, 3rd ed.; Academic Press: Orlando, FL, USA, 1985.

7. Khan, S.; Yasmin, G.; Khan, R.; Hassan, N.A.M. Hermite-based Appell polynomials: Properties and applications. J. Math. Anal. Appl. 2009, 351, 756-764. [CrossRef]

8. Carlitz, L. Degenerate Stiling, Bernoulli and Eulerian numbers. Utilitas Math. 1979, 15, 51-88.

9. Young, P.T. Degenerate Bernoulli polynomials, generalized factorial sums, and their applications. J. Number Theorey 2008, 128 , 738-758. [CrossRef]

10. Cenkci, M.; Howard, F.T. Notes on degenerate numbers. Discrete Math. 2007, 307, 2375-2395. [CrossRef]

11. Ryoo, C.S. Notes on degenerate tangent polynomials. Glob. J. Pure Appl. Math. 2015, 11, 3631-3637.

12. Haroon, H.; Khan, W.A. Degenerate Bernoulli numbers and polynomials associated with degenerate Hermite polynomials. Commun. Korean Math. Soc. 2018, 33, 651-669.

13. Hwang, K.W.; Ryoo, C.S. Some identities involving two-variable partially degenerate Hermite polynomials induced from differential equations and structure of their roots. Mathematics 2020, 8, 632. [CrossRef] 
14. Kim, T.; Kim, D.S.; Kwon, H.I.; Ryoo, C.S. Differential equations associated with Mahler and Sheffer-Mahler polynomials. Nonlinear Funct. Anal. Appl. 2019, 24, 453-462.

15. Ryoo, C.S. A numerical investigation on the structure of the zeros of the degenerate Euler-tangent mixed-type polynomials. J. Nonlinear Sci. Appl. 2017, 10, 4474-4484 [CrossRef]

16. Ryoo, C.S. Differential equations associated with tangent numbers. J. Appl. Math. Inform. 2016, 34, 487-494. [CrossRef]

17. Ryoo, C.S. Some identities involving Hermitt Kampé de Fériet polynomials arising from differential equations and location of their zeros. Mathematics 2019, 7, 23. [CrossRef]

18. Ryoo, C.S.; Agarwal, R.P.; Kang, J.Y. Differential equations associated with Bell-Carlitz polynomials and their zeros. Neural Parallel Sci. Comput. 2016, 24, 453-462. 